\title{
A Geometric Approach to Predicting Bounds of Downstream Model Performance
}

\author{
Brian J. Goode \\ Virginia Tech \\ bjgoode@vt.edu
}

\author{
Debanjan Datta \\ Discovery Analytics Center \\ Virginia Tech \\ ddatta@vt.edu
}

\begin{abstract}
This paper presents the motivation and methodology for including model application criteria into baseline analysis. We will focus on detailing the interplay between the common measures of mean square error (MSE) and accuracy as it relates to perceived model performance. MSE is a common aggregate measure for the performance of predictive regression models. The advantages are numerous. MSE is agnostic to the choice of model given that the set of possible outcome values are defined on the appropriate metric space. In practice, decisions on how to subsequently use a trained model are based on predictive performance, relative to a baseline where input features are not used - colloquially a "random model". However, the relative performance gains of a model in terms of MSE to the baseline does not guarantee commensurate gains when deployed in downstream applications, systems, or processes. This paper demonstrates one derivation of a distribution to qualify MSE performance for multi-class decision making systems desiring a certain level of accuracy. The model error is qualified through comparison to relevant baselines tied to the application suited to evaluating individual outcome performance criteria.
\end{abstract}

\section{CCS CONCEPTS}

- Mathematics of computing $\rightarrow$ Distribution functions; Probabilistic representations; • Computing methodologies $\rightarrow$ Supervised learning.

\section{KEYWORDS}

Machine Learning, Error metrics, Classification, Interpretability, Social systems

\section{ACM Reference Format:}

Brian J. Goode and Debanjan Datta. 2020. A Geometric Approach to Predicting Bounds of Downstream Model Performance. In Proceedings of the 26th ACM SIGKDD Conference on Knowledge Discovery and Data Mining (KDD '20), August 23-27, 2020, Virtual Event, CA, USA. ACM, New York, NY, USA, 9 pages. https://doi.org/10.1145/3394486.3403210

\section{INTRODUCTION}

The ubiquity of application frameworks that incorporate machine learning along with domain knowledge and human input to solve real-world problems motivates a closer look into these systems.

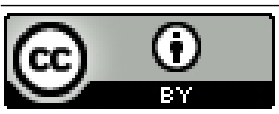

This work is licensed under a Creative Commons Attribution International 4.0 License. KDD '20, August 23-27, 2020, Virtual Event, CA, USA

(C) 2020 Copyright held by the owner/author(s)

ACM ISBN 978-1-4503-7998-4/20/08.

https://doi.org/10.1145/3394486.3403210

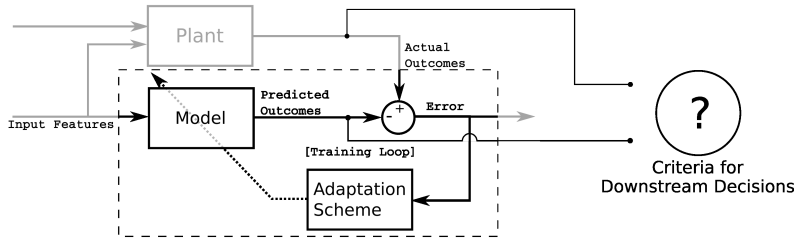

Figure 1: Depiction of a model-system. By incorporating the application into consideration, we can view model performance more broadly in terms of system interactions and more specifically in terms of intended application. This paper adopts this view to motivate developing an accuracy distribution for an application based on MSE during model training.

Interpreting machine learning models in terms of how the features could make sense to the practitioner and help end users has been studied in works such as [19] and [15].

Let us define the integration of model into application or pipeline as a model-system or application framework and is shown in Figure 1 . The term model-system refers to an overall system that takes into account additional criteria of robustness and stability, which are not typically examined from a machine learning model's perspective. The three blocks represent the model being trained, the adaptation scheme based on feedback, and the output stage. The latter can be referred to as the plant, the observed outcome generator, or "reality". For a basic review of control systems theory see [5]). The conventional model development scheme is shown inside the dashed bounding box. Outside of the bounding box are processes that can affect model performance, but may not be directly included in the training or model performance analysis. There exist techniques in (adaptive) control theory to estimate and ensure system stability, but in many ML tasks, the plant is too complex to reasonably estimate or include as part of model training. Research to address plant complexity is ongoing (c.f., abductive looping [7] and estimating emergent behavior [11]). Here, however, we will limit system analysis by focusing on error encoding and harmonizing a desired application with model development.

The subsequent analysis is motivated by these broad research questions:

RQ1. How are errors interpreted contextually in training and in application?

RQ2. How does the application influence the choice of error measure(s) in a model-system?

RQ3. How does one determine whether a model is feasible? 


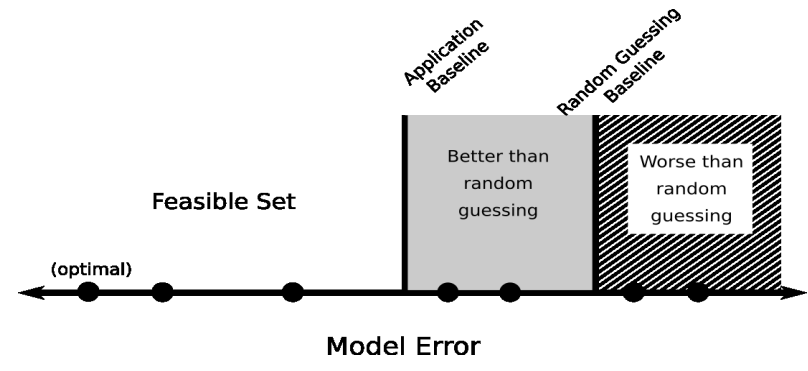

Figure 2: An illustration of the feasible set being determined by baselines. Conventionally, a model error is compared against random guessing from a priori outcomes. However, for a given application, the feasible set may be more restrictive as shown. This paper derives probabilistic (distance) error from qualitative error measures.

These questions are answered in terms of a scenario where a simple model is trained using MSE per competition requirements, which is a probabilistic interpretation of error. Given the goal of the model is to predict continuous values with least error, MSE presents itself as an appropriate loss function during training since it penalizes large residuals. However, we ultimately evaluate the model's performance in a final application scenario using accuracy, which is a qualitative interpretation of error. This scenario consists of properly classifying individual cases, and these cases are consequential. We define consequential as individual case-level error is important regardless of how a model performs in aggregate. Therefore, there is some minimum accuracy requirement on models that can be considered to be feasible, which needs to be translated into MSE terms for model validation. The set of models meeting these requirements is called the feasible set, and as shown in Figure 2, may be more stringent than a random baseline.

The ensuing analysis qualifies MSE by creating an equivalency class of residual vectors corresponding to a particular level of accuracy. Assuming no prior knowledge of the data, a given MSE value for $\mathrm{N}$ observations fully defines an $\mathrm{N}$-dimensional accuracy distribution that will be subsequently derived. It translates to the probability of achieving a level of accuracy given a fixed level of MSE. In the following, a background of work leading to this result will be presented. A derivation of the distribution will be shown using a geometric interpretation of the error. Because of the intractability of solving for the distribution in high dimensions in closed form, two methods of approximating the solution are given. Finally, a motivating example is presented based on prior participation in the Fragile Families Challenge (FFC). The FFC sought to predict six outcomes of social well-being for children and their families at age 15 based on longitudinal data from birth until age 9 . In total, this paper represents one application scenario, but shows the promise of extending the boundary of model performance analysis to a model-system level to better understand, translate, and interpret model errors.

\section{RELATED WORK AND BACKGROUND}

The existing literature on error types and measurement is vast and spans many disciplines [3], [2]. Previous works such as [16] have focused on some other aspects, such as characterization and detection of prediction errors; albeit in a different setting.

This abridged review will only discuss the relevant aspects of error that are important for decision-making with ML model predictions in social systems in this context. The discussion will be limited to the establishment of an upper bound for the feasible set of model errors adequate for a given application. The interpretation and evaluation of the developed or trained model will be in an application scenario. The core of this discourse is the interplay between individual and aggregate error, the interpretation of the error, and exogenous constraints on performance requirements. Using the terminology given in [8], the interpretability of error metrics is categorized as: (i) qualitative metrics, (ii) probabilistic metrics, and (iii) ranking metrics. Qualitative metrics such as accuracy and F-score are based on thresholds and count the number of occurrences of correct or incorrect classifications. Probabilistic metrics such as mean square error (MSE) and cross-entropy error represent aggregated distances from correctly predicting individual or case-level values. Unlike qualitative metrics, probabilistic metrics encode the degree of incorrectness as a measure. Ranking metrics such as AUC will not be considered here, but are useful for recommender systems where the order is important.

The intersection of qualitative and probabilistic metrics is especially acute in classification problems. A classifier that predicts classes with low confidence may be well-calibrated because the probability of predicted classes matches the actual probability of occurrence. However, the ability to discriminate between classes may still be lacking [20]. Prior work has decomposed the MSE (Brier Score) into various components such as reliability, resolution, and uncertainty [17]. The decomposition of MSE in terms of the estimator(model) bias and variance is important as well, and the Bias-Variance tradeoff problem is well studied in machine learning literature [13], [4]. These decompositions are good for ranking models, but still lack a meaningful qualitative explanation. For example, a model with $70 \%$ accuracy may just be predicting one class that happens to occur $70 \%$ of the time. In cases of traditional multi-class classification, where class imbalance exists, simply utilizing F-1 score will result in incorrect interpretation of model performance. One needs to resort to metrics such as micro F-1 or Matthews Correlation Coefficient rather than macro F-1 for correct evaluation of model performance. In the same vein, understanding the applicability of an error metric towards correct evaluation and interpretation thereof, for a model is indispensable part of the process. There have been a number of experimental evaluations into different error metrics for classification models. Specific to MSE and Accuracy, experimental evidence $[8,9]$ shows a high degree of correlation between these two measures.

Error measures such as MSE allow for convex loss functions which are tractable, but qualitative errors such as accuracy are not necessarily convex [21]. In particular, the accuracy loss function for a linear classifier is NP-hard to calculate and a small change in the input features can result in a large change in the loss. Contrarily, normed loss functions such as MSE are convex and can use gradient descent methods to arrive at a solution [1]. A mapping from MSE to accuracy enables training and testing a model using MSE while having the scope of interpretability as accuracy, using thresholds. 
Using a geometric interpretation of the error, this manuscript establishes an equivalency class of accuracy values for a given MSE value. The relationship then becomes a probability distribution over possible accuracy values given a specified MSE.

Two unifying frameworks for interpreting multiple types of error are proper scoring rules and threshold choice methods [12]. The work presented here builds on the previous analyses by directly identifying the relationship between MSE and accuracy, probabilistic and qualitative interpretations, respectively. The correlated behavior of MSE and Accuracy identified in the experimental investigations is made explicit, with corroborating evidence and a rigorous derivation of the relationship. The relationship is constructed using geometric interpretations of the errors, which differs from the previous unifying frameworks that focus on loss functions. Our perspective emphasizes developing error constraints for an application and that this error should be specified a priori. Using an example ML model challenge, we illustrate how such a feasible set can be specified based on an application scenario in mind.

\section{PROBLEM STATEMENT AND FRAMEWORK}

A predictive model, $\mathcal{M}_{\mathcal{P}}: \mathbf{x}_{\mathbf{i}} \mapsto y_{i}$ is a function that takes a vector, $\mathbf{x}_{\mathbf{i}} \in \mathbb{R}^{D}$, as input. This vector, containing $D$ features or predictors, maps to an output or prediction, $y_{i} \in \mathbb{R}$. During training and validation, a set of parameters, $\mathcal{P}$, is selected to minimize a chosen loss function or error over a set of observations $\left(\mathbf{x}_{\mathbf{i}}, y_{i}\right)$. The indices, $i \in\{1,2, \ldots, N\}$ represent $N$ individual observations. For each observation, the difference between the model prediction and the observed or actual measured value is termed the residual:

$$
r_{i}=y_{i}-y_{i}^{*}
$$

Here, $y_{i}^{*}$ is the true value defined by observation or measurement. There is a residual for each observation, and together they form a residual vector, $\mathbf{r} \in \mathbb{R}^{N}$.

An aggregate error measure of the predictive performance of a model, $e: \mathbf{r} \mapsto \mathbb{R}$, is a surjective function that maps residual vectors to point values. The analysis in this article focuses on the mean square error (MSE) of the residuals:

$$
e_{m s e}(\mathbf{r})=\frac{1}{N} \sum_{i=1}^{N} r_{i}^{2}
$$

The relation between aggregate error and individual case level error is defined by the application or how the model will be used. We focus on the case where models trained using MSE are coupled to multi-class decision applications and individual classifications of observations are consequential. This application is described by partitions, $Z_{m} \subset Y$, as a cover of the outcome space $Y \subseteq \mathbb{R}$. The partitions are defined as regions of the outcome space such that

$$
Y=\bigcup_{m=0}^{M} Z_{m} ; Z_{m} \cap Z_{n}=\emptyset, \forall n \neq m
$$

Predictive outcomes $y_{i} \in z_{m}$ will be prescribed a decision value, $m \in \mathbb{N}$. To illustrate the analysis methods, the partitioning scheme is simplified by defining an ordered set of constant $\Delta$-interval outcome partitions

$$
Z_{m}:=\left[a_{m}, a_{m}+\Delta\right) ; Z_{M}:=\left[a_{M}, a_{M}+\Delta\right]
$$

The bounds of each interval in the partition, $a_{m}$, are defined recursively: $a_{m+1}=a_{m}+\Delta$. Specifying $\Delta=\frac{B-A}{M}$ equally partitions the space between upper and lower bounds $A$ and $B$, respectively.

Problem Statement. Consider a uniform $\Delta$-partitioning of the outcome space (Eqn. 4) and a set of true observations that are midpoints of the partitions,

$$
Y^{*}:=\left\{A+\frac{1}{2} \Delta, A+\frac{3}{2} \Delta, \ldots, A+\frac{2 N+1}{2} \Delta\right\} ; A \in \mathbb{R}
$$

Given these outcome specifications, and using the definition of residual in Eqn. 1, a single predicted value $y_{i}$ is paired with a partition, $m$, when

$$
\left|r_{i}\right|=\left|y-y^{*}\right| \leq \frac{\Delta}{2}
$$

The total number of mis-classifications is therefore defined by

$$
e_{\text {class }}(\mathbf{r})=\sum_{i=1}^{N} \mathbb{1}\left(\left|r_{i}\right|>\frac{\Delta}{2}\right)
$$

where $\mathbb{1}(\cdot)$ is the indicator function.

Problem. For an unknown residual vector, $\mathbf{r}$, known MSE error, $e_{m s e}(\mathbf{r})=m, N$ observations, and a $\Delta$-partition of the output space, what is the probability of having inaccuracy $e_{\text {class }}(\mathbf{r})=k$, $P(k \mid m, \Delta)$ ?

\section{ANALYSIS}

The main insight needed to derive the probability of inaccuracy given an MSE value, $P(k \mid m, \Delta)$, is that the probability distribution is defined by the portion of an $(N-1)$-sphere contained within partitions of an $N$-cube. The geometry results by definition of MSE and inaccuracy given in Eqns. 2 and 7, respectively. The probability distribution is derived, and an approximate method suitable for computation is presented in the following two sections.

The $(N-1)$-sphere is defined as the set of points that are equidistant from the origin using distance measure, \|\|$_{2}$

$$
\mathcal{S}^{N-1}(R):=\left\{x \in \mathbb{R}^{N}:\|x\|_{2}=R\right\}
$$

where $R$ is the radius. We immediately see that rearranging the terms of Eqn. 2 and taking the square root produces a function defining MSE in terms of \|\|$_{2}$ distance.

$$
\sqrt{e_{m s e}(\mathbf{r}) N}=\sqrt{\sum_{i=1}^{N} r_{i}^{2}}=\|\mathbf{r}\|_{2}
$$

Using these relations the residual manifold for an MSE value is specified.

Definition 4.1. For a given MSE value, $m$ and $N$ observations, the set of residuals that satisfies $e_{m s e}(\mathbf{r})=m$ is the spherical manifold $\mathcal{S}^{N-1}(R)$ with $R=\sqrt{m \times N}$.

The radius of the hyper-sphere grows with MSE, and the spherical manifold increases dimension with each additional observation.

Individual classification error thresholds over the set of observations are partitions in $\mathbb{R}^{N}$ that form an $N$-dimensional cube. The generic $N$-cube is defined by

$$
C^{N}(a, b):=\left\{\left[a_{i}, b_{i}\right]^{N}: i \in 1,2, \ldots, N\right\}
$$


where $a_{i}, b_{i}$ are bounds for each dimension of the cube. As demonstrated in Eqn. 7, a classification error for a single observation occurs when $\left|r_{i}\right|>\frac{\Delta}{2}$.

Definition 4.2. Consider a 1-cube, $C_{i}^{+}=C^{1}\left(0, \frac{\Delta}{2}\right)$, and its complement $C_{i}^{-}=\mathbb{R}^{+} \backslash C_{i}^{+}$. A classification error does not occur when $\left|r_{i}\right| \in C_{i}^{+}$. A classification error does occur when $\left|r_{i}\right| \in C_{i}^{-}$.

Definition 4.2 for 1 error is used to determine inaccuracy, or count of mis-classifications, as partitions of $\mathbb{R}^{+N}$. For example, in two dimensions, the cube, $\left(C^{+}\right)^{2}=C_{1}^{+} \times C_{2}^{+}$, defines a region in $\mathbb{R}^{2}$ where $|\mathbf{r}| \in\left(C^{+}\right)^{2}$ have 0 classification errors. Similarly, $\left(C^{-}\right)^{2}=C_{1}^{-} \times C_{2}^{-}$, defines a region in $\mathbb{R}^{2}$ where $|\mathbf{r}| \in\left(C^{-}\right)^{2}$ has 2 classification errors. There is one error for each observation. However, the cube $\left(C^{+}\right)\left(C^{-}\right)=C_{1}^{+} \times C_{2}^{-}$has no classification error for the first observation dimension of $|\mathbf{r}|$ and one classification error for the second. In the case of $\left(C^{-}\right)\left(C^{+}\right)=C_{1}^{-} \times C_{2}^{+}$, the classification errors for each dimension are reversed and the cube is located in a different region of the space. We use the notation $\left(\mathrm{C}^{-} \mathrm{C}^{+}\right)_{k}^{N}$ to identify the union of all permutations for the $C^{+} C^{-}$ordering for $k$ occurrences of $C^{-}$in $N$ dimensions. For the two dimensional example,

$$
\begin{aligned}
& \left(C^{-} C^{+}\right)_{0}^{2}=\left(C^{+}\right)^{2} \\
& \left(C^{-} C^{+}\right)_{1}^{2}=\left(C_{1}^{-} \times C_{2}^{+}\right) \cup\left(C_{2}^{-} \times C_{1}^{+}\right) \\
& \left(C^{-} C^{+}\right)_{2}^{2}=\left(C^{-}\right)^{2}
\end{aligned}
$$

For the general case, with $N$ observations and $k$ occurrences of $C^{-}$,

$$
\left(C^{-} C^{+}\right)_{k}^{N}:=\bigcup_{j} C_{s_{j, 1}}^{-} \times \cdots \times C_{s_{j, k}}^{-} \times C_{s_{j, k+1}}^{+} \times \cdots \times C_{s_{j, N}}^{+}
$$

where each $s_{j} ; j \in\left\{1,2, \ldots,\left(\begin{array}{l}N \\ k\end{array}\right)\right\}$ is a permutation set $\{1,2, \ldots, N\}$ such that $C_{s_{j, 1}}^{-} \times \cdots \times C_{s_{j, k}}^{-} \neq C_{s_{l, 1}}^{-} \times \cdots \times C_{s_{l, k}}^{-}, \forall j \neq l$ and $C_{s_{j, 1}}^{+} \times$ $\cdots \times C_{s_{j, k}}^{+} \neq C_{s_{l, 1}}^{+} \times \cdots \times C_{s_{l, k}}^{+}, \forall j \neq l$.

This specific construction and partitioning of the $N$-cube interior makes it possible to define regions of space that correspond to particular values of classification error. Note that we use symmetry about the origin to simplify the geometry of the problem to absolute values of the residual vector and keep the volume of interest in $\mathbb{R}^{+N}$. The aggregate error is then represented as a distance measure from the origin. For MSE, this is the hyper-sphere as defined above. A slice is then formed by taking the intersection of the inscribed hypercube and hyper-sphere. Taking the distribution of classification error over the slice is a geometric realization of the sought after probability. Harder to visualize in greater dimensions, the two dimensional representation of the sets and spatial allocation of errors is given in Figure 3.

Proposition 4.3. Consider the $N$-cube, $\left(C^{-} C^{+}\right)_{k}^{N}$ and residual vectors on the spherical manifold $\mathbf{r} \in \mathcal{S}^{N-1}(R)$. Given an integer $k$, vector $\mathbf{r}$, and definition of classification error in Eqn. 7, then $e_{\text {class }}(\mathbf{r})=$ $k$ iff $|\mathbf{r}| \in \mathcal{S}^{N-1}(R) \cap\left(C^{-} C^{+}\right)_{k}^{N}$.

Proof. Consider Eq. 7, $e_{\text {class }}(\mathbf{r})=\sum_{i=1}^{N} \mathbb{1}\left(\left|r_{i}\right|>\frac{\Delta}{2}\right)$. Using Def. 4.2 , we can then substitute the equivalent set indicator function,

$$
e_{\text {class }}(\mathbf{r})=\sum_{i=1}^{N} \mathbb{1}\left(\left|r_{i}\right| \in C_{i}^{-}\right)
$$

Then, there is some ordering of the residuals, $s_{j}$, such that

$$
e_{\text {class }}(\mathbf{r})=\sum_{i=1}^{\kappa} \mathbb{1}\left(\left|r_{s_{j, i}}\right| \in C_{s_{j, i}}^{-}\right)
$$

where $\kappa<N$. Assume an error value, $k$. The minimum value for $\kappa=k$ so that

$$
k=\sum_{i=1}^{k} \mathbb{1}\left(\left|r_{s_{j, i}}\right| \in C_{s_{j, i}}^{-}\right)
$$

Because $r_{i}>0$ and $C_{i}^{+}=C_{i}^{-} \backslash \mathbb{R}^{+}$, we can then rewrite the equation so that

$$
k=\sum_{i=1}^{k} \mathbb{1}\left(\left|r_{s_{j, i}}\right| \in C_{s_{j, i}}^{-}\right)+\sum_{i=k+1}^{N}\left[\mathbb{1}\left(\left|r_{s_{j, i}}\right| \in C_{s_{j, i}}^{+}\right)-1\right]
$$

Therefore, if each element of $\mathbf{r}$ for any $\mathbf{r} \in \mathcal{S}^{N-1}(R)$ is in each element of the union of $\left(C^{-} C^{+}\right)_{k}^{N}$ in Eq. 14, then $|\mathbf{r}| \in \mathcal{S}^{N-1}(R) \cap$ $\left(C^{-} C^{+}\right)_{k}^{N}$. The inverse deduction is self-evident from the fact that the correct class error is only obtained when the first term exactly contains all of the elements of the residual vector over the $\Delta$-threshold.

In order to quantify the probability, $P(k \mid m, \Delta)$, the size of the intersections of the spherical manifold in each of the cube partitions is calculated using integration. For this, we define the following spherical measure (i.e., "surface area”),

$$
\gamma\left(R, C^{N}\right)=\int_{a_{N}}^{b_{N}} \cdots \int_{a_{1}}^{b_{1}} \mathbb{1}\left(\mathcal{S}^{N-1}(R)\right) d x_{1} \ldots d x_{N}
$$

where $\mathbb{1}(\cdot)$ is the indicator function, and the bounds $a_{i}, b_{i}$ are the 1-cube boundaries defined in Eqn. 10. Inserting Eqn. 14, using the radial symmetry of the sphere, and applying Fubini's theorem, the following result is obtained,

$$
\begin{aligned}
& \gamma\left(R,\left(C^{-} C^{+}\right)_{k}^{N}\right) \\
& =\left(\begin{array}{l}
N \\
k
\end{array}\right) \gamma\left(R, C_{1}^{-} \times \ldots \times C_{k}^{-} \times C_{k+1}^{+} \times \ldots \times C_{N}^{+}\right) \\
& =\left(\begin{array}{l}
N \\
k
\end{array}\right) \int_{0}^{\frac{\Delta}{2}} \cdots \int_{0}^{\frac{\Delta}{2}} \int_{\frac{\Delta}{2}}^{\infty} \cdots \int_{\frac{\Delta}{2}}^{\infty} \mathbb{1}\left(\mathcal{S}^{N-1}(R)\right) d x_{1} \ldots d x_{N}
\end{aligned}
$$

A special case of the spherical measure is when the manifold of residuals is completely inscribed in a hypercube. This will always be true for the cube when the bounds are permitted to go to $\pm \infty$. The cube identified by

$$
C_{\infty}^{N}=[-\infty,+\infty]^{N}
$$

completely inscribes $\mathcal{S}^{N-1}(R)$ for all $R<\infty$ and has the property

$$
\mathcal{S}^{N-1}(R) \cap C_{\infty}^{N}=\mathcal{S}^{N-1}(R)
$$

such that the following equalities hold

$$
\begin{aligned}
\gamma\left(R, C_{\infty}^{N}\right) & =\int_{-\infty}^{\infty} \cdots \int_{-\infty}^{\infty} \mathbb{1}\left(\mathcal{S}^{N-1}(R)\right) d x_{1} \ldots d x_{N} \\
& =2^{N} \int_{0}^{\infty} \cdots \int_{0}^{\infty} \mathbb{1}\left(\mathcal{S}^{N-1}(R)\right) d x_{1} \ldots d x_{N} \\
& =\frac{N \pi^{\frac{N}{2}}}{\Gamma\left(\frac{N}{2}+1\right)}
\end{aligned}
$$




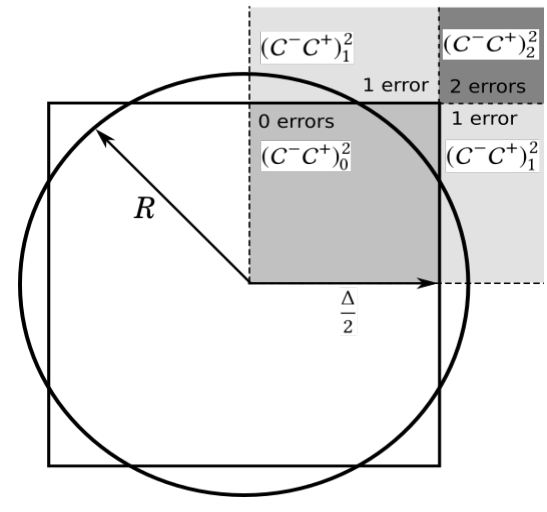

Figure 3: Two dimensional illustration of the sets. Each partition and extension of the hypercube represents regions of one mis-classification, two mis-classifications, etc. Symmetry is used to confine analysis to positive values. The probability of achieving any one of those error counts is determined by the surface area of the $\mathrm{N}$-sphere within each of the regions. In the case of this figure, there is no surface area of the sphere in the 2-error partition, and so has probability 0.

The last formula is the $\mathrm{N}$-dimensional surface area of a hyper-sphere of radius $R$ and corresponds to a the total measure over all of the residuals located on the spherical manifold. With these tools, the main proposition in this paper is given.

Proposition 4.4. For a uniform partitioning of the outcome space $Y$, with separation distance, $\Delta>0$, the set of true values $Y^{*}$ defined by Eqn. 5, MSE value, $m>0$, and number of observations $N>0$, the probability of having $0 \leq k \leq N$ integer classification errors is

$$
P(k \mid m, \Delta)=2^{N}\left[\frac{\gamma\left(R,\left(C^{-} C^{+}\right)_{k}^{N}\right)}{\gamma\left(R, C_{\infty}^{N}\right)}\right]
$$

where $R=\sqrt{m \times N}$.

Proof. The event of interest is the location of a residual vector in a cube partition, $\left(C^{-} C^{+}\right)_{k}^{N}$ and the set of all events, $\mathcal{E}=$ $\mathbb{P}\left(\left\{\left(C^{-} C^{+}\right)_{0}^{N},\left(C^{-} C^{+}\right)_{1}^{N}, \ldots,\left(C^{-} C^{+}\right)_{N}^{N}\right\}\right)$, where $\mathbb{P}()$ denotes the power set, is closed under all set operations. From the definition of the cube, we have that

$$
C_{\infty}^{N}=\bigcup_{k=0}^{N}\left(C^{-} C^{+}\right)_{k}^{N}
$$

and boundaries $\gamma_{\min }=\min _{k}\left[\gamma\left(R,\left(C^{-} C^{+}\right)_{k}^{N}\right)\right]=0$ as the lower and $\gamma_{\max }=\max _{k}\left[\gamma\left(R,\left(C^{-} C^{+}\right)_{k}^{N}\right)\right]=\gamma\left(R,\left(C_{\infty}^{N}\right)\right.$ as the upper bound. Therefore, the denominator is a partition function that normalizes the function so that $0<P(k \mid m, \Delta)<1$. Lastly, we have

$$
P\left(\bigcup_{i} e_{i}\right)=\sum_{i} P\left(e_{i}\right)
$$

for $e \in \mathcal{E}$ and any index $i$, which results from the individual events being disjoint, $\left.\left.\left(C^{-} C^{+}\right)_{j}^{N}\right) \cap\left(C^{-} C^{+}\right)_{k}^{N}\right)=\varnothing ; j \neq k$, and
$\left.\left.\gamma\left(R,\left(C^{-} C^{+}\right)_{j}^{N}\right) \cup\left(C^{-} C^{+}\right)_{k}^{N}\right)\right)=\gamma\left(R,\left(C^{-} C^{+}\right)_{j}^{N}\right)+\gamma\left(R,\left(C^{-} C^{+}\right)_{k}^{N}\right)$.

The main result of this section is Eqn. 26. MSE is cast as a distribution over the number of classification errors in the residuals. This distribution can be thought of in general terms as the "surface area" of the $\mathrm{N}$-sphere inscribed within partitions of $\mathrm{N}$-cubes that cover the entire region of $R^{N}$.

\section{APPROXIMATE SOLUTION}

The result from the previous section established a geometric interpretation of a probability distribution for the number of misclassifications given a specified MSE and classification threshold. However, evaluating the $N$ integrals over the indicator function is not trivial to calculate. Presented here is a method to approximate the solution by first creating a linear combination of Gaussian radial basis functions for small $N$. Because this will break down for large $N$, a second approximation is presented for large $N$ using one Gaussian radial basis function and relaxing the constant MSE with an aggregate error distribution.

The foundation for approximating the solution begins with the exact integral form of the probability in Eqn. 26 is

$$
\begin{aligned}
P(k \mid m, \Delta) & =c \int_{a_{N}}^{b_{N}} \cdots \int_{a_{1}}^{b_{1}} \delta\left(\mathbf{x}^{T} \mathbf{x}-R^{2}\right) d x_{1 \cdots N} \\
a_{i} & = \begin{cases}\frac{\Delta}{2}, & i \leq k \\
0, & \text { otherwise }\end{cases} \\
b_{i} & = \begin{cases}\infty, & i \leq k \\
\frac{\Delta}{2}, & \text { otherwise }\end{cases} \\
R & =\sqrt{m \times N} \\
c & =2^{N}\left(\begin{array}{l}
N \\
k
\end{array}\right) \frac{\Gamma\left(\frac{N}{2}+1\right)}{N \pi^{\frac{N}{2}}}
\end{aligned}
$$

In order to be practical in a variety of applications, the solution to this probability integral needs to be calculated efficiently in very high dimensions. The first step is to cast the dirac delta function in the integral in Eqn. 29 using a Gaussian function. The substitution is

$$
\begin{aligned}
g\left(\mathbf{x}^{T} \mathbf{x}\right) & =\delta\left(\mathbf{x}^{T} \mathbf{x}-R^{2}\right) \\
& =\frac{1}{\epsilon \sqrt{2 \pi}} e^{-\frac{\left(\mathbf{x}^{T} \mathbf{x}-R^{2}\right)^{2}}{2 \epsilon^{2}}}, \epsilon \rightarrow 0
\end{aligned}
$$

and approaches $\delta(\cdot)$ as $\epsilon$ approaches 0 .

The second step is to approximate the new integral term in Eqn. 35 using a linear basis of radially symmetric functions, $F_{j}(\cdot)$ that are separable by product. Such a basis is constructed as

$$
g\left(\mathbf{x}^{T} \mathbf{x}\right) \approx \mathbf{F}\left(\mathbf{x}^{T} \mathbf{x}\right)=\phi_{1} F_{1}\left(\mathbf{x}^{T} \mathbf{x}\right)+\ldots+\phi_{J} F_{J}\left(\mathbf{x}^{T} \mathbf{x}\right)
$$

where the basis functions have the property

$$
F_{j}\left(\mathbf{x}^{T} \mathbf{x}\right)=F_{j}\left(x_{1}^{2}\right) F_{j}\left(x_{2}^{2}\right) \cdots F_{j}\left(x_{N}^{2}\right)
$$

where the $\phi_{j}: j \in\{1 \ldots J\}$ are constants. A suitable choice for a radial basis function is the Gaussian function

$$
F_{j}\left(\mathbf{x}^{T} \mathbf{x}\right)=e^{\frac{-\mathbf{x}^{T} \mathbf{x}}{\sigma_{j}^{2}}}
$$


Because of the product separability of $F_{j}(\cdot)$ in Eqn. 37, then the definite $j^{\text {th }}$ integral of dimension $d$ for constant bounds $a, b$ is defined by

$$
\begin{aligned}
I_{j}^{d}\left(\mathbf{x}^{T} \mathbf{x} ; a, b\right) & =\int_{a}^{b} \cdots \int_{a}^{b} F_{j}\left(\mathbf{x}^{T} \mathbf{x}\right) d x_{1} \cdots d \\
& =\left[\int_{a}^{b} F_{j}\left(x^{2}\right) d x\right]^{d}
\end{aligned}
$$

where $d=\operatorname{dim}(\mathbf{x})$. Substituting Eqn. 38 into Eqn. 40 and completing the $j^{\text {th }}$ integral, we arrive at the specific integral solution

$$
\begin{aligned}
I_{j}^{d}\left(\mathbf{x}^{T} \mathbf{x} ; a, b\right) & =\left[\int_{a}^{b} e^{\frac{-x^{2}}{\sigma_{j}^{2}}} d x\right]^{d} \\
& =\frac{1}{2^{d}} \pi^{\frac{d}{2}} \sigma_{j}^{d}\left[\operatorname{erf}\left(\frac{b}{\sigma_{j}}\right)-\operatorname{erf}\left(\frac{a}{\sigma_{j}}\right)\right]^{d}
\end{aligned}
$$

Using this notation, the probability integral in Eqn. 29 is approximated by

$$
P(k \mid m, \Delta) \approx c \sum_{j} \phi_{j} I_{j}^{k}\left(\mathbf{x}^{T} \mathbf{x} ; \frac{\Delta}{2}, \infty\right) I_{j}^{N-k}\left(\mathbf{x}^{T} \mathbf{x} ; 0, \frac{\Delta}{2}\right)
$$

The gamma term in the numerator is going to magnify any approximation errors of the summands, especially as $N$ increases. To reduce this effect, the surface area term can also be approximated as follows

$$
\begin{aligned}
\frac{N \pi^{\frac{N}{2}}}{\Gamma\left(\frac{N}{2}+1\right)} & \approx \sum_{j} \phi_{j} I_{j}^{N}\left(\mathbf{x}^{T} \mathbf{x} ;-\infty, \infty\right) \\
& \approx 2^{N} \sum_{j} \phi_{j} I_{j}^{N}\left(\mathbf{x}^{T} \mathbf{x} ; 0, \infty\right)
\end{aligned}
$$

Inserting this into Eqn. 43, the final approximation is given as

$$
P(k \mid m, \Delta) \approx\left(\begin{array}{c}
N \\
k
\end{array}\right) \frac{\sum_{j} \phi_{j} I_{j}^{k}\left(\mathbf{x}^{T} \mathbf{x} ; \frac{\Delta}{2}, \infty\right) I_{j}^{N-k}\left(\mathbf{x}^{T} \mathbf{x} ; 0, \frac{\Delta}{2}\right)}{\sum_{j} \phi_{j} I_{j}^{N}\left(\mathbf{x}^{T} \mathbf{x} ; 0, \infty\right)}
$$

By substituting in the solution to the definite integral in Eqn. 42 and rearranging the constant terms a simpler version becomes

$$
P(k \mid m, \Delta) \approx\left(\begin{array}{l}
N \\
k
\end{array}\right) \frac{\sum_{j} \phi_{j}\left[1-\operatorname{erf}\left(\frac{\Delta}{2 \sigma_{j}}\right)\right]^{k}\left[\operatorname{erf}\left(\frac{\Delta}{2 \sigma_{j}}\right)\right]^{N-k}}{\sum_{j} \phi_{j}}
$$

Finally, the probability can be cast as a sum of binomial distributions, $\mathcal{B}()$, such that

$$
\begin{aligned}
P(k \mid m, \Delta) & \approx \frac{\sum_{j} \phi_{j}\left(\begin{array}{c}
N \\
k
\end{array}\right)\left(p_{j}\right)^{k}\left(1-p_{j}\right)^{N-k}}{\sum_{j} \phi_{j}} \\
& \approx \frac{\sum_{j} \phi_{j} \mathcal{B}\left(k, N, p_{j}\right)}{\sum_{j} \phi_{j}}
\end{aligned}
$$

where the $j^{\text {th }}$ term of

$$
p_{j}=1-\operatorname{erf}\left(\frac{\Delta}{2 \sigma_{j}}\right)
$$

In general, Eqn. 50 is only suitable for small $N$. As $N$ increases, weights $\phi_{j}$ for smaller $\sigma_{j}$ become exponentially small, and the largest $\sigma_{j}$ will have an disproportionately large impact on the approximation.

There are many factors that will affect the outcome of this choice of approximation. First, choosing a Gaussian kernel to approximate the delta function introduces a quadratic term in the exponential. Because radial basis functions are chosen to simplify the integration to multiplication in a variable number of dimensions, the quadratic term in the exponential needs to be approximated by a linear sum of Gaussian functions. Any error will be magnified by the positive exponential term in the delta function approximation expansion. At present, with some trial and error, a simple linear regression is used to determine the weights given a set of variance terms.

\section{Approximation for large samples, $N$.}

We now relax the requirement that the MSE, $m$, is known precisely and constant. With this relaxation, the sum square of the residuals (SSR) in the residual vector is no longer a constant radius, $R$, but a stochastic variable $r$. We will specify a distribution where $P(r=$ $R) \rightarrow 1$ as $N \rightarrow \infty$. We will define $r$ with the following probability distribution

$$
P(r)=A\left(r^{N}\right) e^{-\frac{1}{\sigma_{1}^{2}}\left(r^{2}\right)}
$$

where $A$ is a constant normalization factor. An interpretation of this choice of distribution is that the sum square of residuals (SSR) follow the chi-square distribution

$$
\frac{1}{\sigma_{1}^{2}} \sum_{i=0}^{N} r_{i}^{2} \sim \chi_{N}^{2}
$$

For sufficiently large $N$, we can use the fact that the mass of the Gaussian function converges to radius $R$ so that samples drawn from the distribution satisfy $\|\mathbf{x}\| \rightarrow R$ if $\sigma_{1}=\sqrt{\frac{2 R}{N}}$ in Eqn. 50 (see [14]). In this case, we only need one Gaussian approximation and can set $\phi_{1}=1$ and $\phi_{j \neq 1}=0$.

Proposition 5.1. The probability of $k$ mis-classifications with threshold $\triangle$ for expected MSE $E(m)$ where residual vectors are distributed with radii $P(r)$ in Eqn. 51 is

$$
P(k \mid E(m), \Delta)=\mathcal{B}(k, N, p)
$$

where $p=1-\operatorname{erf}\left(\frac{\Delta}{2 \sqrt{\frac{2 R}{N}}}\right)$.

Proof. Consider the derivative

$$
\left.\frac{d P(r)}{d r}\right|_{R}=e^{\frac{-R^{2}}{\sigma_{1}^{2}}}\left[N R^{N-1}-\frac{2 R^{N+1}}{\sigma_{1}^{2}}\right]
$$

When set to 0 , the following equality holds

$$
\frac{N \sigma_{1}^{2}}{2}=R^{2}
$$

Setting $\sigma_{1}=\sqrt{\frac{2 R}{N}}$ results convergence in $P(r=R) \rightarrow R$. As $N$ grows, the variance term decreases while the maximum value remains at $r=R$ as enforced by the first derivative. Therefore, only one term is needed in Eqn. 50 with $\sigma_{1}=\sqrt{\frac{2 R}{N}}$ and the summation collapses to one binomial distribution. 


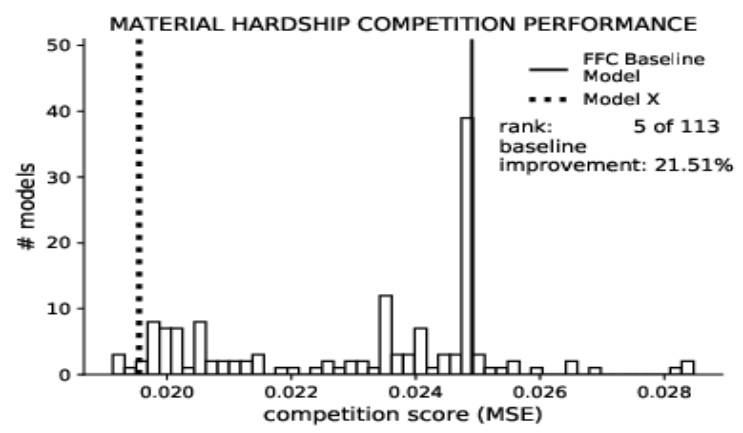

Figure 4: Material Hardship performance in the Fragile Families Challenge using a probabilistic interpretation of error (MSE). The histograms shows the performance of all of the models in the Challenge including the mean baseline.

This result has a number of interesting properties. First is the connection between accuracy $\left(1-\frac{k}{N}\right)$ and MSE through the binomial distribution. The approximation weakens the requirements of precisely specifying the MSE to vastly simplify and avoid having to solve the $N$ exact integrals. And, as $N$ grows, the approximation converges following $\sqrt{\frac{2 R}{N}}$. Therefore, one can use this distribution to estimate expected accuracy for large observations. Crucially, independence is not assumed between observations enabling higher level group effects to be accounted for in the accuracy estimate from an aggregate measure.

Finally, none of these techniques require data to be available at the time of analysis. We note this, because traditional baselines stem from a priori estimates of available data (e.g., mean value guess). Here, the modeler must constrain the threshold, MSE, or accuracy value. Once specified, the other values can be determined, without needing data. Therefore, a baseline for MSE derived from a threshold and accuracy specification is application driven based on desired policy or use.

\section{CASE STUDY}

In this section, we analyze the performance of a model with respect to interpretability and use in an application scenario. In the first case viz. RQ1 and RQ2, we explore how the choice of application impacts the metric used. For RQ3, we describe a feasible set of models identified by performance criteria.

Using the distribution outlined previously, the feasible set will be defined for the Fragile Families Challenge $(F F C)$ that occurred during the summer of 2016 [6]. Based on the Fragile Families and Child Wellbeing Study (FFCWS) [18], the FFC challenged participants to create predictive models for six outcome variables related to individual children and their families. The outcome variables were GPA, Grit, Material Hardship, Job Training, Layoff, and Eviction. The training data consisted of over 12,000 features, sourced from surveys, interviews, and objective tests. Over a hundred participants submitted, and evaluation was performed using a test set. Models were ranked based on MSE or Brier Score. We refer to our model as Model X, and is fully reproducible [10].

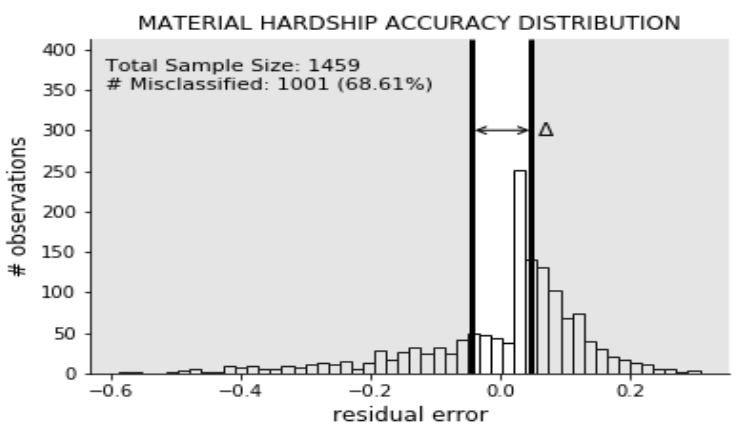

Figure 5: Material Hardship performance in the Fragile Families Challenge using a qualitative interpretation of error (Accuracy). This histogram shows the residuals for Model X only.

To better focus on the methodological issues, we will only discuss the Material Hardship outcome variable. In the $F F C$, this score was an average of responses to 11 binary (0-1) questions. This means that the Material Hardship outcome is effectively ordinal with a set of possible values $\{0, .09,0.18, \ldots, 1.0\}$. Each response variable is implicitly categorical, but is standardized to be in $[0,1]$. Importantly, this operation is extrinsic to the model, and a part of the application system within which the model is used. The outcome of the Material Hardship category in the Challenge is shown in the histogram in Figure 4. MSE values are displayed on the $\mathrm{x}$-axis and the number of models in the MSE range are on the $y$-axis. The MSE values appear to be quite low, but MSE inherently does not have meaning without a relative context to compare against. The baseline shown in the figure and that used during the FFC was the expected value of the outcome variable. Although baseline has multiple contextual meanings, here we mean one that does not use any input features. In this comparison, Model $X$ contends favorably amongst the competing models and random baseline.

Changing the context for RQ1, let us consider a model-system error where the Material Hardship model will be used to adjudicate individual, case-level, decisions. This system necessitates the definition of thresholds or class boundaries. Using the definition of Material Hardship, the process by which it is calculated, and assuming that a model is to be both well calibrated and discriminating among the classes, a natural choice of threshold would be \pm 0.045 . Each of the possible outcomes of Material Hardship are treated as bin centroids. The accuracy of the model in this system is displayed in Figure 5. Immediately, we see that by qualifying the mean square error with accuracy based on the definition of Material Hardship the accuracy performance is approximately $32 \%$.

It is evident with respect to RQ2 that the interpretation of the two approaches to error necessitates a choice of application. In the former, MSE shows that the model outputs are better calibrated than the baseline model. However, this is mostly because of a penalty on the ability of the model to discriminate between two classes. The mean baseline model is restricted to predicting the set of ordinal values, and the submission is able to make predictions that lie in between the ordinal classes. Even with little utility from the 
features, merely choosing a value in between two of the most frequent ordinal values will result in less error than reported by the mean baseline. In applications where calibration is important, and results are considered in aggregate then the MSE is the appropriate measure. One would interpret the model as well calibrated, but not very discriminating. On the contrary, if one were interested in model performance at the level of individual observations, then the accuracy error reveals that the model is not very capable of properly classifying the Material Hardship classes as defined.

Visiting RQ3, we establish the requirements for a feasible set for model error. MSE is a suitably chosen and convenient error metric for evaluation in many cases; however this may be at times without due rigor. For training and validation in our model-system, MSE is chosen as well. To establish a feasible set per the modelsystem above, we identify a qualitative constraint on the number of mis-classifications to be tolerated. For example, let us assume that we will put the Material Hardship model into service as a predictor of at risk families in terms of material well-being. It will then be used to suggest one of several interventions based on the predicted score. In practice, this might translate into execution of real world actions depending on observation bins. For this example, it suffices to specify a number of equally spaced bins over the range of potential outcomes. We will stick with the original partitioning above by setting the threshold to 0.045 .

Using the distribution in Eqn. 47, the expected accuracy of the model is shown as a function of MSE in Figure 6. The probabilities were generated by setting $N=1459$, which corresponds to the number of training samples that were ultimately used by the model [10]. The figure captures a number of items related to the interplay of accuracy and MSE. First, the relationship between accuracy (qualitative error) and MSE (probabilistic error) is clearly seen to be monotonically decreasing as would be expected. However, the expected accuracy (solid black line) does not follow a linear relationship with accuracy over the entire range of accuracy values as found in the experimental evidence $[8,9]$. In this analysis, the correlation coefficient over this range is around 0.3. Both cited experimental investigations reveal a correlation coefficient of around 0.9. However, if the range of correlation is restricted to MSE values between .002 and 1.3, then we do approach the correlation coefficients reported previously. Therefore, we see that there is an accuracy sensitivity dependence on the domain of the MSE values. Accuracy values near $100 \%$ show diminishing returns to MSE improvement, as do accuracy values near $0 \%$. In terms of the example, the marker shown in 6 that demonstrates the model error and the current location of the mean baseline error are located in a region that is sensitive to adjustments in MSE.

Secondly, Eqn. 47 defines a probability distribution over accuracy, but also gives a floor for the minimum accuracy. As MSE is decreased, there is still a chance that one can perform better in terms of accuracy but with lower probability. The only cap on accuracy occurs when $R>\sqrt{N} \frac{\Delta}{2}$, and there is guaranteed to be at least one error over the set of observations. This becomes asymptotically insignificant with increasing $N$. Therefore, if a baseline is established to define a feasible set for model error, there is a guarantee on the minimum accuracy associated with that error. At present, our proposed model for the $F F C$, is being applied in a setting where the aforementioned baseline establishes an effective minimum of around $32 \%$ accuracy. However, if a particular application of this model required a minimum accuracy of $80 \%$, then the MSE criterion would be set almost an order of magnitude lower.

By specifying a model-system including the intended application, constraints are effectively added to the original baseline model to create the feasible set. We may start with a data driven baseline with sufficient capacity to capture the distribution of data (e.g. a deep neural network), and subject it to constraints on the lower bound on the desired downstream model performance (i.e., caselevel accuracy). This constraint can be determined a priori before data is in hand, helping establish a non-random baseline. For a deployed system, this baseline can very well be a SoTA model.

\section{CONCLUSION}

This article presents a probability distribution that relates MSE to interpreted accuracy using a geometric interpretation. In its current form, the derivation assumes a uniform partitioning of continuous error into equal sized bins. These bins can represent specific policy groupings or interventions to be placed on observations having a certain amount of error, or they can be multi-classification outcomes that are the result of possible outcomes themselves (e.g., when the outcome domain has less resolution than the prediction domain). The result is a direct relation of the probabilistic interpretation of error (MSE) with a qualitative interpretation of model performance (accuracy). As discussed through the example, the expected value of accuracy given a MSE value tends to corroborate past experiments relating the two values, but only within a specific interval.

The utility of our approach lies in that different types of error are directly related so that predictions of performance can be made to establish a feasible set of model error without data. By considering the "final mile" of how a model will be used and the requirements of an application, downstream performance criteria can be folded into the error analysis at training even if the errors are not coincident. Using the distribution in this paper, one can state a criterion for model usage qualitatively based on the needs of an application, but train and rank models using MSE. In short, one can train a model on aggregate error, but establish a baseline that has certain guarantees for individual prediction error. This is anticipated to allow for better error translation in fields concerned with applications in policy and decision making. As motivated in the example, an immediate consequence of this approach is that traditional baselines of a prior distributions of outcomes on training data (e.g., mean baseline) can be made consonant with intended applications to establish a feasible set. Firstly our work proposes to connect different types of errors typically involve an objective like loss, utility, or risk. Secondly, we show how choice of error is integral to framework design. Models may not always be standalone entities to train or optimized, but the bound of analysis need to be broadened to consider how the operations around evaluating them translate to application. Thirdly, and specific to this distribution and multi-class definition of policy, the converse problem can also be solved where one model may establish what is possible given its error and become a component of policy design. Finally, the integration methods estimate the partitioning of an $\mathrm{N}$-sphere inscribed in an $\mathrm{N}$-cube. These can be extended to more general partitions of the $\mathrm{N}$-sphere, but will not collapse nicely 


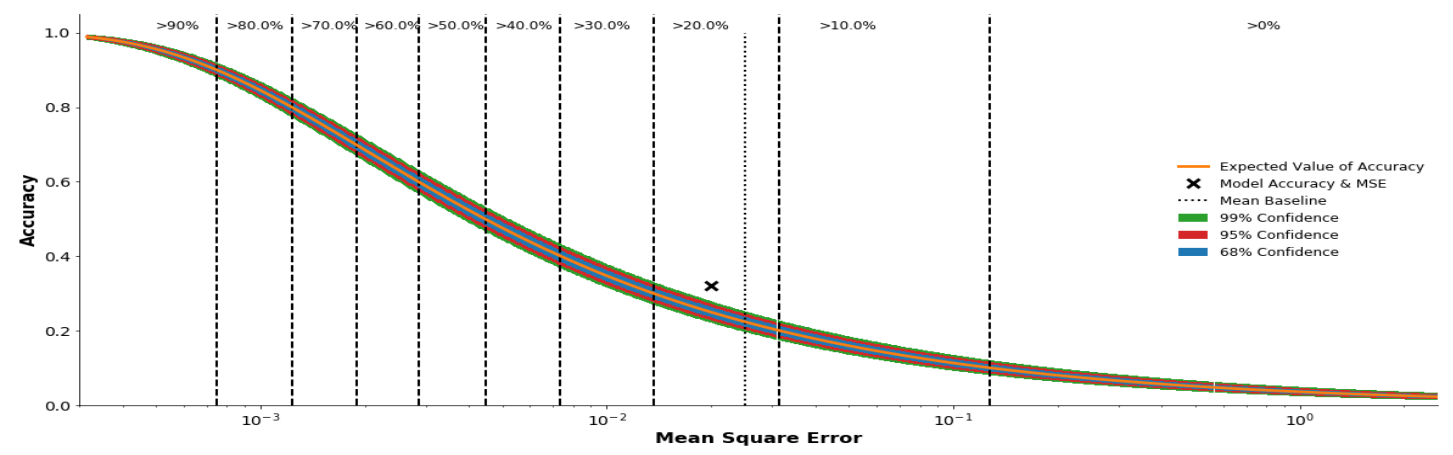

Figure 6: This figure is a concise representation of how a criterion for MSE can be selected to define the feasible set of models based on the probability distribution of qualitative error (Accuracy). The solid black line and the gray shadings around it indicate the relationship between MSE and Accuracy when $N=1459$. In the case of the example, there were 1459 training observations. The solid black line is the expected value of $E[P(k \mid E(m), \Delta)]$. The gray shadings are $68 \%$, 95\%, and 99\% confidence intervals around the expected value. The MSE continuum has been separated into regions of minimum accuracy as indicated by the vertical dashed lines. MSE values occurring in these intervals are guaranteed to have the minimum stated accuracy value along the top of the chart. The dotted vertical line indicates the mean baseline MSE value. It's intersection with the probability curves indicates the likely accuracy values to be seen. In this case, the mean baseline is expected to have a floor of $30 \%$ accuracy and a likely accuracy of about $31 \%$. The realized training error and accuracy of the Material Hardship model is shown by the tick mark. The increased accuracy performance is outside of the "expected" range, but the initial range does not take into account class imbalances. Using this figure, one can establish a new performance baseline to establish the feasible set based on the minimum accuracy desired. For example, if we wanted to obtain a minimum expected accuracy of $80 \%$, then our target MSE should be set closer to $\mathbf{0 . 0 0 2 5}$, or an order of magnitude lower than the mean baseline.

into the binomial form seen here. More broad reaching implications also follow from the approach, but need further consideration and motivates future research.

\section{ACKNOWLEDGMENTS}

Funding for the Fragile Families and Child Wellbeing Study was provided by the Eunice Kennedy Shriver National Institute of Child Health and Human Development through grants R01HD36916, R01HD39135, and R01HD40421 and by a consortium of private foundations, including the Robert Wood Johnson Foundation. Funding for the FFC was provided by the Russell Sage Foundation.

\section{REFERENCES}

[1] Yoshua Bengio, Nicolas L Roux, Pascal Vincent, Olivier Delalleau, and Patrice Marcotte. 2006. Convex neural networks. In Advances in neural information processing systems (18 ed.). MIT Press, Cambridge, 123-130.

[2] Rich Caruana and Alexandru Niculescu-Mizil. 2006. An empirical comparison of supervised learning algorithms. In Proceedings of the 23rd international conference on Machine learning. Association for Computing Machinery, New York, NY, USA 161-168.

[3] R Dennis Cook and Sanford Weisberg. 1982. Residuals and influence in regression New York: Chapman and Hall, New York, NY, USA.

[4] Pedro Domingos. 2000. A unified bias-variance decomposition for zero-one and squared loss. AAAI/IAAI 2000 (2000), 564-569.

[5] Richard C. Dorf and Robert H. Bishop. 2000. Modern Control Systems (9th ed.) Prentice-Hall, Inc., Upper Saddle River, NJ, USA.

[6] Matthew J. Salganik et al. 2020. Measuring the predictability of life outcomes with a scientific mass collaboration. Proceedings of the National Academy of Sciences 117, 15 (2020), 8398-8403. https://doi.org/10.1073/pnas.1915006117

[7] Y. Ren et al. 2018. Generative Modeling of Human Behavior and Social Interactions Using Abductive Analysis. In 2018 IEEE/ACM Int. Conf. on Adv in Soc. Networks Analysis and Mining. IEEE, Barcelona, 413-420. https://doi.org/10.1109/ASONAM. 2018.8508282
[8] C. Ferri, J. Hernández-Orallo, and R. Modroiu. 2009. An Experimental Comparison of Performance Measures for Classification. Pattern Recogn. Lett. 30, 1 (Jan. 2009), 27-38. https://doi.org/10.1016/j.patrec.2008.08.010

[9] Lisa Gaudette and Nathalie Japkowicz. 2009. Evaluation Methods for Ordinal Classification. In Advances in Artificial Intelligence, 22nd Canadian Conference on Artificial Intelligence, Canadian AI 2009, Kelowna, Canada, May 25-27, 2009, Proceedings. Springer Berlin Heidelberg, Berlin, Heidelberg, 207-210. https: //doi.org/10.1007/978-3-642-01818-3 25

[10] Brian J. Goode, Debanjan Datta, and Naren Ramakrishnan. 2019. Imputing Data for the Fragile Families Challenge: Identifying Similar Survey Questions with Semiautomated Methods. Socius 5 (2019).

[11] Brian J. Goode and Bianica Pires. 2019. Training and Application Error for Decision Models. Journal of Policy \& Complex Systems 5, 2 (2019), 165-180.

[12] José Hernández-Orallo, Peter Flach, and Cèsar Ferri. 2012. A Unified View of Performance Metrics: Translating Threshold Choice into Expected Classification Loss. F. Mach. Learn. Res. 13, 1 (Oct. 2012), 2813-2869. http://dl.acm.org/citation. $\mathrm{cfm}$ ?id=2503308.2503332

[13] Gareth James, Daniela Witten, Trevor Hastie, and Robert Tibshirani. 2013. An introduction to statistical learning. Vol. 112. Springer, New York, NY, USA.

[14] W. Krauth. 2006. Statistical Mechanics: Algorithms and Computations. Oxford University Press, Oxford.

[15] Scott M Lundberg and Su-In Lee. 2017. A unified approach to interpreting model predictions. In Advances in Neural Information Processing Systems. Curran Associates, Inc., Red Hook, NY, USA, 4765-4774.

[16] Christopher Meek. 2016. A characterization of prediction errors.

[17] Allan H. Murphy. 1973. A New Vector Partition of the Probability Score. Fournal of Applied Meteorology (1962-1982) 12, 4 (1973), 595-600. http://www.jstor.org/ stable/26176769

[18] Nancy E. Reichman, Julien O.Teitler, Irwin Garfinkel, and Sara S.McLanahan. 2001. Fragile Families: sample and design. Children and Youth Services Review 23, 4 (2001), 303-326. https://doi.org/10.1016/S0190-7409(01)00141-4

[19] Marco Tulio Ribeiro, Sameer Singh, and Carlos Guestrin. 2016. Why should i trust you?: Explaining the predictions of any classifier. , 1135-1144 pages.

[20] Philip E. Tetlock. 2006. Expert Political fudgement: How Good Is It? How Can We Know? Princeton University Press, Princeton, New Jersey.

[21] Paul Tseng. 2010. Approximation accuracy, gradient methods, and error bound for structured convex optimization. Mathematical Programming 125, 2 (01 Oct 2010), 263-295. https://doi.org/10.1007/s10107-010-0394-2 